\title{
Efficient Quantum Key Distribution With Trines Of Reference-Frame-Free Qubits
}

\author{
Gelo TABiA ${ }^{1,2}$ and Berthold-Georg EnGlerT ${ }^{3,4}$ \\ 1 Department of Physics and Astronomy, University of Waterloo, Waterloo, Ontario, Canada N2L 3G1 \\ 2 Perimeter Institute, Waterloo, Ontario, Canada N2L $2 Y 5$ \\ 3 Centre for Quantum Technologies, National University of Singapore, Singapore 117543 \\ 4 Department of Physics, National University of Singapore, Singapore 117542
}

PACS 03.67.Dd - Quantum cryptography and communication security

PACS 03.67.Hk - Quantum communication

PACS 03.67.-a - Quantum information

\begin{abstract}
We propose a rotationally-invariant quantum key distribution scheme that uses a pair of orthogonal qubit trines, realized as mixed states of three physical qubits. The measurement outcomes do not depend on how Alice and Bob choose their individual reference frames. The efficient key generation by two-way communication produces two independent raw keys, a bit key and a trit key. For a noiseless channel, Alice and Bob get a total of 0.573 key bits per trine state sent (98\% of the Shannon limit). This exceeds by a considerable amount the yield of standard trine schemes, which ideally attain half a key bit per trine state. Eavesdropping introduces an $\epsilon$-fraction of unbiased noise, ensured by twirling if necessary. The security analysis reveals an asymmetry in Eve's conditioned ancillas for Alice and Bob resulting from their inequivalent roles in the key generation. Upon simplifying the analysis by a plausible symmetry assumption, we find that a secret key can be generated if the noise is below the threshold set by $\epsilon=0.197$.
\end{abstract}

Introduction. - Mutually non-orthogonal quantum states are important in quantum key distribution (QKD) because such states cannot be completely distinguished from each other and hence they are intentionally used to transmit classical information while preventing eavesdropping. For qubits, the qubit trine represents the smallest complete set of non-orthogonal states. Earlier trine-based protocols include the schemes by Bechmann-Pasquinucci and Peres [1] and by Phoenix, Barnett, and Chefles [2].

When QKD is performed, it is generally assumed that Alice and Bob share a common reference frame, the precise nature of which depends on the specific information carriers involved. For instance, correct orientation of Alice and Bob's coordinates is necessary for proper alignment of the preparation and measurement apparatus. Practical implementations of cryptographic protocols require establishing a rigid shared frame in advance or a frequent automatic realignment. A lack of a shared reference frame is equivalent to the presence of decoherence in the quantum channel [3].

In this contribution, we describe a trine-based cryptographic protocol that uses reference-frame-free qubits and a novel scheme for key generation. We report the asymp- totic noise threshold below which this QKD procedure is secure. The security analysis involves a very plausible, yet unproven, simplifying symmetry assumption.

Basics of trine schemes. - A qubit trine is represented by a symmetric set of three states lying in the $X Z$-plane of the Bloch sphere, where adjacent vectors are separated by $120^{\circ}$; see fig. 1. Let us call the qubit trine $T=\{|A\rangle,|B\rangle,|C\rangle\}$. Alice prepares her qubits in any one of the trine states with equal probability, and sends these qubits one at a time to Bob. Bob measures the qubits he receives with a probability operator measurement (POM) whose outcomes are not projectors to the trine $T$, such as $|A\rangle\langle A|$ but rather to states orthogonal to $T$, i.e., states belonging to the set $T^{\prime}=\left\{\left|A^{\prime}\right\rangle,\left|B^{\prime}\right\rangle,\left|C^{\prime}\right\rangle\right\}$, where

$$
\left|\left\langle A \mid A^{\prime}\right\rangle\right|^{2}=0, \quad\left|\left\langle A \mid B^{\prime}\right\rangle\right|^{2}=\left|\left\langle A \mid C^{\prime}\right\rangle\right|^{2}=\frac{3}{4},
$$

with analogous relations holding for $|B\rangle$ and $|C\rangle$. Since Alice works only with the trine $T$ while Bob is concerned only with states from the complementary trine $T^{\prime}$, we can simplify matters by treating corresponding states of $T$ and $T^{\prime}$ as identical. For example, if Alice sends $A$, we say Bob 


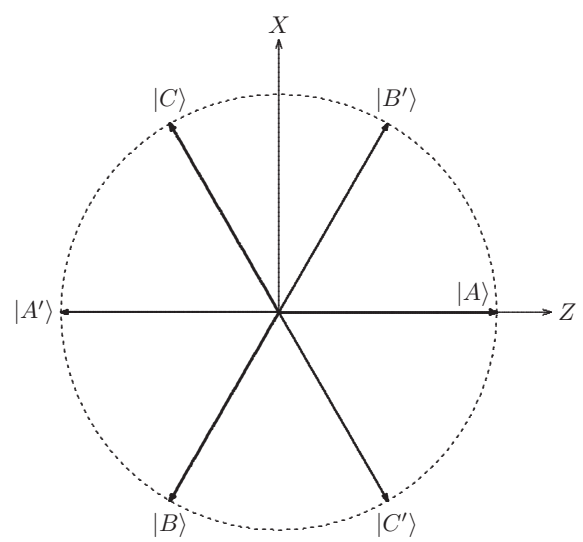

Fig. 1: Qubit trine $T$ and complementary trine $T^{\prime}$ in the Bloch representation. The projectors on the respective kets are all symbolized by vectors in the $X Z$-plane.

never measures $A$ but has equal probability of obtaining either $B$ or $C$. In this description, the joint probabilities of the quantum communication channel are given by table 1 . for which

$$
I(A: B)=\log _{2} \frac{3}{2}=0.585
$$

is the mutual information $I(A: B)$ between Alice and Bob. With such a noiseless trine channel, then, they can generate up to 0.585 secret key bits per qubit sent.

The double trine scheme. - If Alice and Bob use physical qubits for the logical qubits of the trine, they must be sure to agree on the coordinates for preparing and measuring their quantum signals. However, they can skip this problem altogether by using reference-frame-free (RFF) qubits. In our scheme a logical qubit is constructed by coupling three physical qubits. For concreteness, we consider the physical qubits to be spin- $\frac{1}{2}$ particles (with $|0\rangle$ for spin-up and $|1\rangle$ for spin-down) and combine their angular momenta in the appropriate manner.

Following the recipe of ref. [4], we consider two trines. In the subspace $\left(j=\frac{1}{2}, m=\frac{1}{2}\right)$ we have the trine

$$
\begin{aligned}
& \left|p_{1}\right\rangle=(|001\rangle-|010\rangle) / \sqrt{2}, \\
& \left|p_{2}\right\rangle=(|100\rangle-|001\rangle) / \sqrt{2}, \\
& \left|p_{3}\right\rangle=(|010\rangle-|100\rangle) / \sqrt{2},
\end{aligned}
$$

and the states

$$
\begin{aligned}
& \left|q_{1}\right\rangle=(|101\rangle-|110\rangle) / \sqrt{2}, \\
& \left|q_{2}\right\rangle=(|110\rangle-|011\rangle) / \sqrt{2}, \\
& \left|q_{3}\right\rangle=(|011\rangle-|101\rangle) / \sqrt{2},
\end{aligned}
$$

\begin{tabular}{|c|c|c|c|c|}
\hline & \multicolumn{3}{|c|}{ Bob } \\
\hline & & A & B & C \\
\hline & A & 0 & $\frac{1}{a}$ & $\underline{1}$ \\
\hline & & ] & & 6 \\
\hline$\stackrel{\Xi}{\sharp}$ & B & $\frac{1}{6}$ & 0 & $\frac{1}{6}$ \\
\hline & $\mathrm{C}$ & $\frac{1}{6}$ & $\frac{1}{6}$ & 0 \\
\hline
\end{tabular}

constitute the trine in the subspace $\left(j=\frac{1}{2}, m=-\frac{1}{2}\right)$. All relevant states are in the $j=\frac{1}{2}$ sector of the three spin- $\frac{1}{2}$ atoms and, for the sake of simplifying the notation, we shall consistently ignore the empty $j=\frac{3}{2}$ sector.
Table 1: Joint probabilities for the noiseless trine channel.

The sums of the projectors to corresponding $p$ - and $q$ states are

$$
W_{i}=\left|p_{i}\right\rangle\left\langle p_{i}|+| q_{i}\right\rangle\left\langle q_{i}\right|=S_{j k},
$$

where $S_{j k}$ projects on the singlet sector for atoms $j$ and $k$, and the indices $i j k$ pertain to all cyclic permutations of 123 . By construction, the $W_{i}$ s are rotationally invariant and hence have the same properties for all reference frames - they are RFF operators. We note that the $W_{i}$ s have two eigenvalues 0 and two eigenvalues 1 , so that $W_{i}$ and $1-W_{i}$ project on orthogonal two-dimensional subspaces, and

$$
\sum_{i=1}^{3} W_{i}=\frac{3}{2}, \quad \operatorname{tr}\left\{W_{i}\right\}=2, \quad \operatorname{tr}\left\{W_{i} W_{j}\right\}=\frac{3 \delta_{i j}+1}{2}
$$

are identities that will be relevant in what follows.

Because our scheme has two independent sets of trines, we call it the double trine scheme. It works as follows. Alice sends a random sequence of the states $\rho_{i}=\frac{1}{2} W_{i}$ to Bob, with the three states occurring with equal frequency, and Bob measures them with a POM whose outcomes are $\Pi_{j}=\frac{2}{3}\left(1-W_{j}\right)$. The resulting joint probabilities,

$$
p_{i j}=\frac{1}{3} \operatorname{tr}\left\{\rho_{i} \Pi_{j}\right\}=\frac{1-\delta_{i j}}{6}
$$

are those of table 1

Signal and idler qubit. - The sum of the three $p$-kets of (3) vanishes - they are linearly dependent because the $j=m=\frac{1}{2}$ sector is two-dimensional. A pair $|++\rangle,|-+\rangle$ of orthogonal kets is identified by

$$
\left(\left|p_{1}\right\rangle,\left|p_{2}\right\rangle,\left|p_{3}\right\rangle\right)=(|++\rangle,|-+\rangle) \frac{1}{\sqrt{2}}\left(\begin{array}{ccc}
1 & \omega & \omega^{2} \\
1 & \omega^{2} & \omega
\end{array}\right),
$$

where $\omega=\exp (\mathrm{i} 2 \pi / 3)$, and likewise we have

$$
\left(\left|q_{1}\right\rangle,\left|q_{2}\right\rangle,\left|q_{3}\right\rangle\right)=(|+-\rangle,|--\rangle) \frac{1}{\sqrt{2}}\left(\begin{array}{ccc}
1 & \omega & \omega^{2} \\
1 & \omega^{2} & \omega
\end{array}\right)
$$

for the $q$-states. We regard the four orthogonal states $| \pm \pm\rangle$ that span the $j=\frac{1}{2}$ sectors of the three spin- $\frac{1}{2}$ atoms as two-qubits states [4] whereby, for example, ket $|+-\rangle$ has 
the signal qubit in the '+' state and the idler qubit in the '-' state.

The signal states $|A\rangle,|B\rangle,|C\rangle$ that we identify as

$$
(|A\rangle,|B\rangle,|C\rangle)=(|+\rangle,|-\rangle) \frac{1}{\sqrt{2}}\left(\begin{array}{ccc}
1 & \omega & \omega^{2} \\
1 & \omega^{2} & \omega
\end{array}\right)
$$

form the single-qubit trine that matters. Upon denoting the Pauli operators of the signal qubit by $X, Y$, and $Z$ and identifying $| \pm\rangle$ with the eigenkets of $Y=\mathrm{i} X Z$, the signal-qubit trine is in the $X Z$ plane as depicted in fig. 1 .

In view of

$$
W_{1}=|A\rangle\left\langle A\left|\otimes 1, \quad W_{2}=\right| B\right\rangle\left\langle B\left|\otimes 1, \quad W_{3}=\right| C\right\rangle\langle C| \otimes 1,
$$

the idler sector is completely irrelevant: Alice encodes the information in the signal qubit only, and Bob's POM does not probe the idler qubit at all. The sole purpose of the idler qubit is to render possible the construction of the rotationally invariant signal qubit. We can, therefore, think of the double trine scheme as a generic scheme of the kind described in the context of fig. 1 with the signal qubit carrying the quantum state from Alice to Bob.

Common source scenario. - Rather than having Alice prepare qubits in the trine states and send them to Bob, who then analyzes them with the trine POM, we can generate the joint probabilities of table 1 in a more symmetric and largely equivalent way. In this alternative scenario, a source distributes entangled two-qubit states to Alice and Bob.

Ideally, the two signal qubits are in their singlet state that is described by the statistical operator

$$
\rho_{0}=|s\rangle\langle s| \quad \text { with }|s\rangle=\frac{|+-\rangle-|-+\rangle}{\sqrt{2}} .
$$

On their respective qubits, Alice and Bob then both measure the same trine POM with the outcomes

$$
\Pi_{i}=|i\rangle \frac{2}{3}\langle i| \text { for } i=A, B, C .
$$

Indeed, the resulting joint probabilities,

$$
p_{j k}=\operatorname{tr}\left\{\Pi_{j} \otimes \Pi_{k} \rho_{0}\right\} \quad \text { for } j, k=A, B, C,
$$

are those of table 1

In the security analysis below, we shall assume that the source is controlled by eavesdropper Eve. Her activities will introduce noise into the quantum channel between Alice and Bob, but they are only accepting qubits from a source that looks like the singlet of (12) with an admixture of unbiased noise,

$$
\rho_{\epsilon}=|s\rangle(1-\epsilon)\langle s|+\frac{\epsilon}{4}
$$

\begin{tabular}{|c|c|c|c|c|}
\hline & \multicolumn{3}{|c|}{ Bob } \\
\hline & & A & B & $\mathrm{C}$ \\
\hline \multirow{6}{*}{$\stackrel{\circlearrowright}{.}$} & \multirow{2}{*}{ A } & $\epsilon$ & $3-\epsilon$ & $3-\epsilon$ \\
\hline & & $\overline{9}$ & 18 & 18 \\
\hline & \multirow{2}{*}{ B } & $\underline{3-\epsilon}$ & $\underline{\epsilon}$ & $3-\epsilon$ \\
\hline & & 18 & $\overline{9}$ & 18 \\
\hline & \multirow{2}{*}{$\mathrm{C}$} & $3-\epsilon$ & $3-\epsilon$ & $\epsilon$ \\
\hline & & 18 & 18 & $\overline{9}$ \\
\hline
\end{tabular}

with $0 \leq \epsilon \leq 1$.

As far as Alice and Bob are concerned, the noise parameter $\epsilon$ characterizes the channel. In the presence of noise,
Table 2: Joint probabilities for the noisy trine channel.

they observe errors in the trine channel: sometimes they get the same measurement outcome for a particular qubit pair, which does not happen in the noise-free case. Rather than the noise-free joint probabilities of table 1, they now have the probabilities of table 2 .

But since their measurements yield only these nine joint probabilities, Alice and Bob cannot determine all fifteen parameters that specify the two-qubit state distributed by the source. In this respect, the trine schemes are markedly different from tomographic protocols [5], such as the sixstate protocol [6] or the Singapore protocol [7], in which full tomography of the source state is central.

If Alice and Bob do not see the symmetric probability table 2, they enforce the symmetry by twirling. For this purpose, they carry out random bilateral rotations on the qubits that leave the singlet component intact while removing any bias from the noise.

Efficient generation of the raw dual key. - Once Alice and Bob finish collecting and measuring their qubits, they get a paired record of measurement results. Next, they communicate over an authenticated public channel to discuss the raw data and distill a cryptographic key. Here we describe a new key generation method that yields mutual information between Alice and Bob closer to the Shannon limit for a trine-based channel [8]. We illustrate the procedure with the sample results shown in table 3 .

To begin, Alice chooses two time slots in a specified order where her outcomes are different. Suppose she selects columns 2 and 5 in table 3 . Alice's pair of letters in these positions is CB. She tells Bob to look at his record at those two particular time slots and he finds he has A in both. He declares he has the same letter in both positions. Alice quickly determines this letter to be A since it is the only result consistent with the expected outcomes for a trine protocol. Both record A for the key. Because there are three possibilities in this scenario, we call this the trit key. Alice and Bob discard the used time slots.

There is another situation to consider. Say for the next round, Alice chooses columns 1 and 4. Bob finds BC for these time slots and announces the following: Record 0 for $\mathrm{BC}$ and 1 for $\mathrm{CB}$. Since Alice has AB, she infers that Bob must have $\mathrm{BC}$, and both of them record 0 for the key. In this case, there are two possibilities, so we call it the bit key. Note that the order of the time slots selected matters: 
Table 3: Example of measurement records for Alice and Bob.

$\begin{array}{llllllll} & 1 & 2 & 3 & 4 & 5 & 6 & 7 \\ \text { Alice } & \text { A } & \text { C } & \text { C } & \text { B } & \text { B } & \text { A } & \text { C } \\ \text { Bob } & \text { B } & \text { A } & \text { A } & \text { C } & \text { A } & \text { C } & \text { B }\end{array}$

they would both record 1 if Alice reversed the order.

The two situations - the trit case and the bit caseare mutually exclusive events so the bit and trit keys are independently built up from the raw data. In the noiseless case, the trit case happens $\frac{1}{4}$ of the time while the bit case happens in the remaining $\frac{3}{4}$. It follows that the number of key bits, per qubit exchanged, that Alice and Bob share in the key sequences thus generated is given by

$$
I(A: B)=\frac{1}{2}\left(\frac{1}{4} \log _{2} 3+\frac{3}{4} \log _{2} 2\right)=0.573,
$$

which is $98 \%$ of the Shannon limit in (2).

Noise in the channel leads to errors in the shared keys, since the unexpected result of getting the same letter during transmission will sometimes occur. The probability that the next letter pair contributes an entry to the trit key is now $p_{\text {trit }}=\frac{1}{12}(3-\epsilon)(1+\epsilon)$, and the probability of contributing to the bit key is $p_{\text {bit }}=\frac{1}{12}\left[(3-\epsilon)^{2}+4 \epsilon\right]$.

In the trit case, the correctly matched pairs in the key (that is, both Alice and Bob write down the same letter, whether A, B, or C) each have probability $(3-\epsilon) /(9+9 \epsilon)$; the other six outcomes where they disagree have probability $2 \epsilon /(9+9 \epsilon)$ each. Likewise in the bit case, the two instances when Alice and Bob agree both have probability $\frac{1}{2}(3-\epsilon)^{2} /\left[(3-\epsilon)^{2}+4 \epsilon\right]$, while for the other two where they disagree the probability is $2 \epsilon /\left[(3-\epsilon)^{2}+4 \epsilon\right]$ each.

These probabilities yield

$$
\begin{aligned}
I_{\text {trit }}(A: B)= & \frac{3-\epsilon}{3+3 \epsilon} \log _{2} \frac{3-\epsilon}{1+\epsilon} \\
& +\frac{4 \epsilon}{3+3 \epsilon} \log _{2} \frac{2 \epsilon}{1+\epsilon} \\
I_{\text {bit }}(A: B)= & \frac{(3-\epsilon)^{2}}{(3-\epsilon)^{2}+4 \epsilon} \log _{2} \frac{2(3-\epsilon)^{2}}{(3-\epsilon)^{2}+4 \epsilon} \\
& +\frac{4 \epsilon}{(3-\epsilon)^{2}+4 \epsilon} \log _{2} \frac{8 \epsilon}{(3-\epsilon)^{2}+4 \epsilon}
\end{aligned}
$$

for the resulting mutual information between Alice and Bob for the two key sequences. For $\epsilon=0.1$, their weighted sum $\frac{1}{2}\left(p_{\text {trit }} I_{\text {trit }}+p_{\text {bit }} I_{\text {bit }}\right)$ equals $96.4 \%$ of the Shannon limit, the mutual information of the joint probabilities in table 2. As functions of $\epsilon, I_{\mathrm{bit}}(A: B)$ and $I_{\text {trit }}(A: B)$ are the monotonously decreasing curves in figs. 2 and 3 below, respectively.

Security analysis. - Eve is given full control of the source and is allowed to keep a quantum record, encoded in ancilla states, of what is sent. We write the source state in the form

$$
|S\rangle=\left|++E_{1}\right\rangle+\left|+-E_{2}\right\rangle+\left|-+E_{3}\right\rangle+\left|--E_{4}\right\rangle,
$$

where, for example, $\left|+-E_{2}\right\rangle$ is the ' + ' state of (10) for Alice's signal qubit, the '-' state for Bob's, and Eve's ancilla in state $\left|E_{2}\right\rangle$. When Alice's POM gives the $j$ th outcome, and Bob's the $k$ th, the reduced ancilla state is described by $\left|E_{j k}\right\rangle$ where $j$ and $k$ independently take on values of $A, B$, or $C$. After accounting for the coefficients in (10) and (13), we have

$$
\left|E_{j k}\right\rangle=\left(\left|E_{1}\right\rangle,\left|E_{2}\right\rangle,\left|E_{3}\right\rangle,\left|E_{4}\right\rangle\right) \frac{1}{3}\left(\begin{array}{c}
\omega^{-j-k} \\
\omega^{-j+k} \\
\omega^{j-k} \\
\omega^{j+k}
\end{array}\right)
$$

with $A B C \widehat{=} 012$ for the $j$ and $k$ values in the exponents.

The joint probabilities of table 2 impose the constraints

$$
p_{j k}=\left\langle E_{j k} \mid E_{j k}\right\rangle=\frac{\epsilon}{9} \delta_{j k}+\frac{3-\epsilon}{18}\left(1-\delta_{j k}\right),
$$

which in turn imply

$$
\begin{aligned}
& \left\langle E_{1} \mid E_{1}\right\rangle+\left\langle E_{2} \mid E_{2}\right\rangle+\left\langle E_{3} \mid E_{3}\right\rangle+\left\langle E_{4} \mid E_{4}\right\rangle=1, \\
& \left\langle E_{1} \mid E_{2}\right\rangle+\left\langle E_{3} \mid E_{4}\right\rangle=0=\left\langle E_{1} \mid E_{3}\right\rangle+\left\langle E_{2} \mid E_{4}\right\rangle, \\
& \left\langle E_{1} \mid E_{4}\right\rangle=0, \quad\left\langle E_{2} \mid E_{3}\right\rangle=-(1-\epsilon) / 2 .
\end{aligned}
$$

These determine nine of the 16 real parameters that specify the positive $4 \times 4$ matrix of the $\left\langle E_{j} \mid E_{k}\right\rangle$ amplitudes.

A convenient choice of the remaining seven real parameters is given by representing the kets $\left|E_{1}\right\rangle, \ldots,\left|E_{4}\right\rangle$ by the columns of a matrix of the form [9]

$$
V=\left(\begin{array}{cccc}
a_{1} & \lambda a_{2} & -\mu a_{2} & 0 \\
0 & r_{1} \cos \theta & -r_{2} \mathrm{e}^{\mathrm{i} \phi} \sin \theta & 0 \\
0 & r_{1} \mathrm{e}^{-\mathrm{i} \phi} \sin \theta & -r_{2} \cos \theta & 0 \\
0 & \mu^{*} a_{1} & -\lambda^{*} a_{1} & a_{2}
\end{array}\right)
$$

where $a_{1}, a_{2}, r_{1}, r_{2}, \phi, \theta$ are real and $\lambda, \mu$ are complex, and their values are subject to

$$
\begin{aligned}
\left(1+|\lambda|^{2}+|\mu|^{2}\right)\left(a_{1}^{2}+a_{2}^{2}\right)+r_{1}^{2}+r_{2}^{2} & =1, \\
\lambda^{*} \mu\left(a_{1}^{2}+a_{2}^{2}\right)+r_{1} r_{2} \mathrm{e}^{\mathrm{i} \phi} \sin (2 \theta) & =\frac{1-\epsilon}{2} .
\end{aligned}
$$

As demonstrated by

$$
\begin{aligned}
& a_{1}=a_{2}=\frac{1}{2} \sqrt{\epsilon}, \quad r_{1}=r_{2}=\frac{1}{2} \sqrt{2-\epsilon}, \quad \lambda=\mu=0 \\
& \phi=0, \quad \sin (2 \theta)=\frac{2-2 \epsilon}{2-\epsilon},
\end{aligned}
$$

for which Alice and Bob's reduced two-qubit state is $\rho_{\epsilon}$ of (15), there surely are permissible values, but it is not obvious which set of parameters is optimal for Eve. With the aid of (19), each permissible $V$ matrix gives us valid column representations for the $\left|E_{j k}\right\rangle \mathrm{s}$.

In fact, Eve is not interested in distinguishing the $\left|E_{j k}\right\rangle$ states themselves but rather the two-ancilla states that are associated with symbols in the key sequences, whereby the bit and trit cases need to be considered separately. 
In the bit case, the two-ancilla state conditioned on Alice concluding that Bob has the letter sequence ' $j k$ ' is given by

$$
\begin{aligned}
\rho_{j k}^{(A)} \propto & \left|E_{k j} E_{l k}\right\rangle\left\langle E_{k j} E_{l k}|+| E_{l j} E_{j k}\right\rangle\left\langle E_{l j} E_{j k}\right| \\
& +\left|E_{k j} E_{j k}\right\rangle\left\langle E_{k j} E_{j k}|+| E_{k k} E_{l j}\right\rangle\left\langle E_{k k} E_{l j}\right| \\
& +\left|E_{l k} E_{j j}\right\rangle\left\langle E_{l k} E_{j j}|+| E_{k k} E_{j j}\right\rangle\left\langle E_{k k} E_{j j}\right|,
\end{aligned}
$$

where $j k l$ can be any permutation of $A B C$. The first three terms account for the cases in which Alice and Bob record the same bit value, and the bit errors are covered by the last three terms. For example, the first term is for the situation when Alice has ' $k l$ ' and Bob has ' $j k$ ' while both have ' $k j$ ' for the last term. Eve has to tell $\rho_{j k}^{(A)}$ and $\rho_{k j}^{(A)}$ apart when Bob announces that his letters are ' $j$ ' and ' $k$ '.

Analogously, in the trit case, we have the conditioned two-ancilla state

$$
\begin{aligned}
\rho_{j}^{(A)} \propto & \left|E_{k j} E_{l j}\right\rangle\left\langle E_{k j} E_{l j}|+| E_{l j} E_{k j}\right\rangle\left\langle E_{l j} E_{k j}\right| \\
& +\left|E_{k k} E_{l k}\right\rangle\left\langle E_{k k} E_{l k}|+| E_{l k} E_{k k}\right\rangle\left\langle E_{l k} E_{k k}\right| \\
& +\left|E_{k l} E_{l l}\right\rangle\left\langle E_{k l} E_{l l}|+| E_{l l} E_{k l}\right\rangle\left\langle E_{l l} E_{k l}\right|
\end{aligned}
$$

when Alice concludes that Bob has letter ' $j$ ' twice, with the first two terms accounting for a correct assignment and the remaining four terms for errors. Here, too, $j k l$ is a permutation of $A B C$, and Eve need to distinguish the three states $\rho_{A}^{(A)}, \rho_{B}^{(A)}$, and $\rho_{C}^{(A)}$.

The six $\rho_{j k}^{(A)}$ s and three $\rho_{j}^{(A)}$ s account for 54 of the 81 two-ancilla kets $\left|E_{j k} E_{j^{\prime} k^{\prime}}\right\rangle$. This is as it should be because the remaining 27 kets are those for which Alice has the same letter twice, and this situation does not occur.

If Eve eavesdrops on Bob, the conditioned two-ancilla states are different. In the bit case we have

$$
\begin{aligned}
\rho_{j k}^{(B)} \propto & \left|E_{k j} E_{l k}\right\rangle\left\langle E_{k j} E_{l k}|+| E_{l j} E_{j k}\right\rangle\left\langle E_{l j} E_{j k}\right| \\
& +\left|E_{k j} E_{j k}\right\rangle\left\langle E_{k j} E_{j k}|+| E_{l j} E_{k k}\right\rangle\left\langle E_{l j} E_{k k}\right| \\
& +\left|E_{j j} E_{l k}\right\rangle\left\langle E_{j j} E_{l k}|+| E_{j j} E_{k k}\right\rangle\left\langle E_{j j} E_{k k}\right|,
\end{aligned}
$$

and the states

$$
\begin{aligned}
\rho_{j}^{(B)} \propto & \left|E_{k j} E_{l j}\right\rangle\left\langle E_{k j} E_{l j}|+| E_{l j} E_{k j}\right\rangle\left\langle E_{l j} E_{k j}\right| \\
& +\left|E_{j j} E_{l j}\right\rangle\left\langle E_{j j} E_{l j}|+| E_{l j} E_{j j}\right\rangle\left\langle E_{l j} E_{j j}\right| \\
& +\left|E_{j j} E_{k j}\right\rangle\left\langle E_{j j} E_{k j}|+| E_{k j} E_{j j}\right\rangle\left\langle E_{k j} E_{j j}\right|
\end{aligned}
$$

apply in the trit case. They differ from their respective counterparts by the error terms. Therefore, we explore both sets of ancilla states to see whether Eve gains any advantage by eavesdropping on either Alice or Bob, or if it does not make any difference to the optimal amount of information she can obtain.

With the assignment of signal-qubit Pauli operators $X$, $Y, Z$ discussed above in the context of (10), the two-qubit state $\rho_{A B}$ that the source distributes to Alice and Bob is specified by the eight fixed expectations values

$$
\begin{aligned}
& \left\langle X_{A}\right\rangle=\left\langle Z_{A}\right\rangle=\left\langle X_{B}\right\rangle=\left\langle Z_{B}\right\rangle=0, \\
& \left\langle X_{A} X_{B}\right\rangle=\left\langle Z_{A} Z_{B}\right\rangle=-(1-\epsilon), \\
& \left\langle X_{A} Z_{B}\right\rangle=\left\langle Z_{A} X_{B}\right\rangle=0
\end{aligned}
$$

together with the seven adjustable expectation values

$$
\begin{aligned}
& \frac{1}{2}\left(\left\langle Y_{A}\right\rangle \pm\left\langle Y_{B}\right\rangle\right)=\left\{\begin{array}{l}
a_{1}^{2}-a_{2}^{2}, \\
r_{1}^{2}-r_{2}^{2}-\left(|\lambda|^{2}-|\mu|^{2}\right)\left(a_{1}^{2}-a_{2}^{2}\right),
\end{array}\right. \\
& \left\langle Y_{A} Z_{B}\right\rangle+\mathrm{i}\left\langle Y_{A} X_{B}\right\rangle=4 \lambda a_{1} a_{2}, \\
& \left\langle Z_{A} Y_{B}\right\rangle+\mathrm{i}\left\langle X_{A} Y_{B}\right\rangle=-4 \mu a_{1} a_{2}, \\
& \left\langle Y_{A} Y_{B}\right\rangle=2\left(a_{1}^{2}+a_{2}^{2}\right)-1,
\end{aligned}
$$

which reveal the physical significance of the seven free parameters in (22). Alice and Bob cannot distinguish between $\rho_{A B}, X_{A} X_{B} \rho_{A B} X_{A} X_{B}, Y_{A} Y_{B} \rho_{A B} Y_{A} Y_{B}$, and $Z_{A} Z_{B} \rho_{A B} Z_{A} Z_{B}$, and Eve gets the same amount of information from the corresponding four sets of conditioned ancilla states. It follows that Eve can just as well choose the parameters in (22) such that $\rho_{A B}=X_{A} X_{B} \rho_{A B} X_{A} X_{B}=$ $Z_{A} Z_{B} \rho_{A B} Z_{A} Z_{B}$. Then, the six expectation values in (30) that involve a single $Y$ vanish, which happens for

$$
a_{1}=a_{2}, \quad r_{1}=r_{2}, \quad \lambda=\mu=0 .
$$

Indeed, it is plausible, and supported by much numerical evidence, that a parameter choice that yields such a particularly noisy $\rho_{A B}$ is advantageous for Eve because then the entanglement between her ancilla and the qubits for Alice and Bob is particularly strong.

With (31), matrix $V$ takes on the simple one-parameter form

$$
V=\frac{1}{2}\left(\begin{array}{cccc}
\sqrt{c} & 0 & 0 & 0 \\
0 & x & -y & 0 \\
0 & y & -x & 0 \\
0 & 0 & 0 & \sqrt{c}
\end{array}\right)
$$

with $0 \leq c \leq 2 \epsilon$ and $x \pm y=\sqrt{2-c \pm 2(1-\epsilon)}$. We return to (24) for $c=\epsilon$, while $c=2 \epsilon$ and $c=2 \epsilon-\epsilon^{2}$ give the $\rho_{A B} \mathrm{~S}$ with minimal concurrence and maximal entropy, respectively; the $\rho_{A B} \mathrm{~s}$ for $2 \epsilon+c \geq 2$ are separable [10].

The following observation lends additional support to (31) and (32): The resulting conditioned ancilla states are such that it does not matter which letter pairs ' $j k$ ' and ' $k j$ ' are to be distinguished in the bit case, or which letter ' $j$ ' is the actual one in the trit case. Eve does not acquire better knowledge about a subset of key entries at the price of knowing less about other subsets. By contrast, such an asymmetry in her knowledge is typically the case if some of the single- $Y$ expectation values in (30) are nonzero.

Accepting thus the hypothesis that it suffices to consider matrices $V$ of the single-parameter form (32), we take the resulting two-ancilla kets $\left|E_{j k}\right\rangle$ and calculate the Holevo-Schumacher-Westmoreland (HSW) bounds [11,12] on $I(A: E)$ and $I(B: E)$ as a function of $c$. After optimizing the value of $c$ for the given value of the noise parameter $\epsilon$, we obtain the monotonically increasing curves in figs. 2 and 3 for the bit key and the trit key, respectively.

The $\epsilon$ values for which these curves intersect the curves representing the corresponding $I(A: B)$ of (17) determine the noise thresholds below which Alice and Bob can generate a secret key from the raw key by the usual procedures 


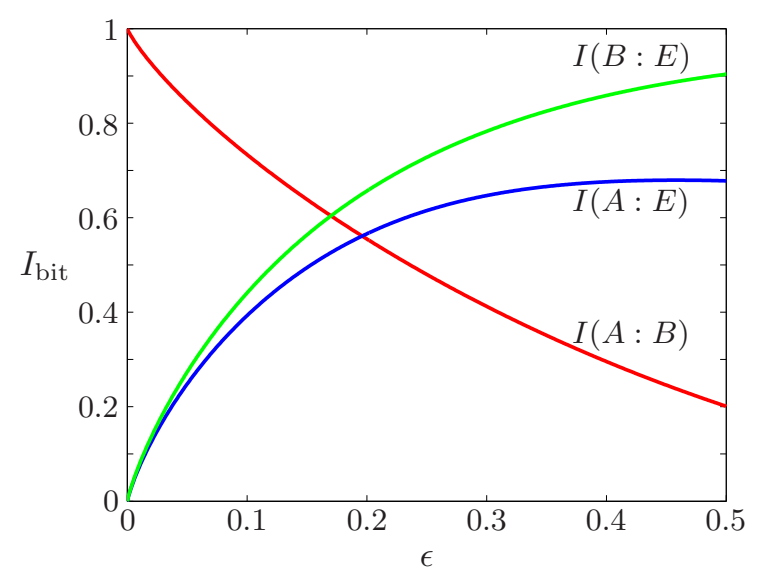

Fig. 2: Optimizing the one-parameter source state: Wiretapper bound for Eve eavesdropping on the bit key. For Alice, the noise threshold is $\epsilon=0.197$, where $I_{\mathrm{bit}}=0.560$. The corresponding numbers for Bob are $\epsilon=0.170, I_{\mathrm{bit}}=0.603$.

of error correction and privacy amplification [13]. Both in the bit case and in the trit case, the thresholds are higher when Eve is eavesdropping on Alice than on Bob. We could not find lower thresholds with any parameter values not restricted by the symmetry requirements (31).

Summary and discussion. - We described a basisindependent trine protocol for QKD that uses RFF signal qubits encoded in mixed states of three physical qubits. The protocol exploits a novel efficient key generation scheme that yields a dual alphabet key. We analyzed the security with a plausible symmetry assumption that simplifies the task to the optimization of a single parameter. As a consequence of the asymmetric roles played by them during the key generation, there are different noise thresholds for eavesdropping on Alice and Bob.

The raw keys need to be processed before Alice and Bob share a secret key. For the error correction and the privacy amplification one of the raw keys serves as the error-free reference, and we choose Alice's key for this purpose because then the higher thresholds apply. We conclude that a secret key can be generated for $\epsilon<0.197$, and one should stay well below this threshold to have a good key bit rate.

Regarding practical implementations of the scheme, we note that the production of entangled states is no routine matter, with the difficulty increasing rapidly with size. A practical system with a common source for Alice and Bob requires six entangled physical qubits for each transmission. It is easier to use the variant where Alice prepares the states and sends them to Bob as this requires only three qubits. As with other QKD protocols, photon polarization is the most likely candidate for the physical qubits. Alice could prepare three-photon trine states by first preparing two of the three photons in a Bell state and the third photon with random polarization; it is possible to achieve this by beginning with an entangled four-photon state and measuring the polarization of the fourth photon

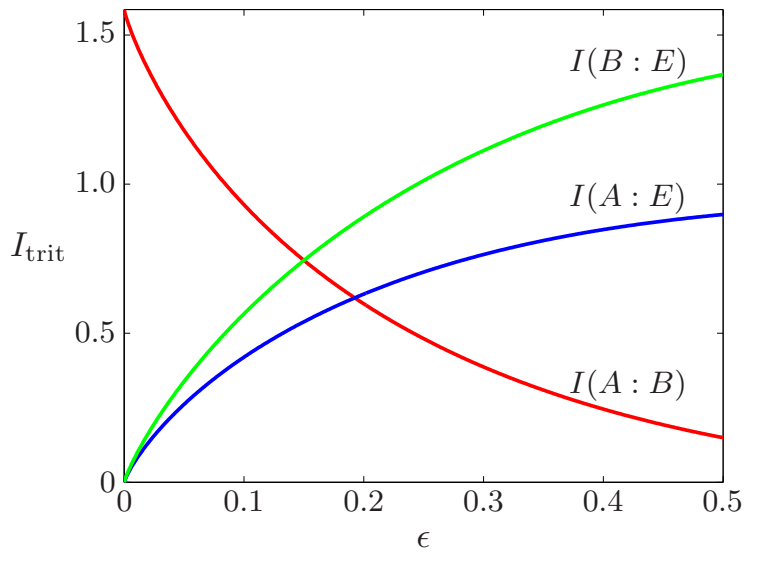

Fig. 3: Optimizing the one-parameter source state: Wiretapper bound for Eve eavesdropping on the trit key. For Alice, the noise threshold is $\epsilon=0.193$, where $I_{\text {trit }}=0.618$. The corresponding numbers for Bob are $\epsilon=0.150, I_{\text {trit }}=0.744$.

with a suitable POM. Bob's POM would then test if one of the three orthogonal Bell states is present for every trio of photons received from Alice. Given the limited efficiency of typical photodetectors, efficient detection of all three photons is a challenge though.

$$
* * *
$$

We are grateful for useful discussions with Jun Suzuki, Syed M. Assad, and Valerio Scarani. BGE thanks Hans Briegel for the kind hospitality in Innsbruck where part of this work was done. Centre for Quantum Technologies is a Research Centre for Excellence funded by the Ministry of Education and National Research Foundation of Singapore.

\section{REFERENCES}

[1] Bechmann-Pasquinucci H. and Peres A., Phys. Rev. Lett., 85 (2000) 3313.

[2] Phoenix S., Barnett A. and Chefles J., J. Mod. Optics, 47 (2000) 507.

[3] Bartlett S., Rudolph T. and Spekkens R., Rev. Mod. Phys., 79 (2007) 555.

[4] Suzuki J., Tabia G. and Englert B.-G., Phys. Rev. A, 78 (2008) 052328.

[5] Liang Y.C. et al., Phys. Rev. A, 68 (2003) 022324.

[6] Bruss D. and Macchiavello C., Phys. Rev. Lett., 88 (2002) 127901.

[7] ENGLert B.-G. et al., arXiv:quant-ph/0412075 (2004).

[8] We owe the idea of the key generation method to W.K. Chua.

[9] Tabia G., M.Sc. Thesis (Singapore, 2009).

[10] Assad S.M., Suzuki J. and Englert B.-G., Int. J. Quant. Inform., 4 (2006) 1003-1012.

[11] Holevo A. S., Probl. Peredachi Inf., 9 (1973) 177.

[12] Schumacher B. and Westmoreland M., Phys. Rev. A, 56 (1997) 131-137.

[13] Renner R., Int. J. Quant. Inform., 6 (2008) 1. 